\title{
A Highly Efficient On-chip 3D Plasmonic Nanofocusing Structure
}

Myung-Ki Kim ${ }^{1}$ and Hyuck Choo ${ }^{1}$

${ }^{1}$ The Moore Laboratory, Electrical Engineering, California Institute of Technology, Pasadena, CA 91125, U.S.A.

\begin{abstract}
We demonstrate and analyze a highly efficient on-chip 3D metal-insulator-metal (MIM) nanofocusing structure. Here, we show the in-depth theoretical design, analysis and discussion to provide a detailed picture of the highly efficient, on-chip nanofocusing process which is linearly tapered in 3D.
\end{abstract}

\section{INTRODUCTION}

Extreme field enhancement using an efficient nanofocusing process is essential for maximizing the performance of nanoscale optical/photonic devices, especially on-chip communication nano-devices [1-3]. However, due to the diffraction limits of the conventional dielectric devices, achieving extreme light-concentrations in the deep sub-wavelength spatial scale has been challenging. The utilization of surface plasmon polaritons (SPPs) has been proposed as a feasible mechanism for overcoming the diffraction limits of conventional focusing devices. However, due to the challenges involved in various loss mechanisms and on-chip nanofabrication, most studies have been limited to the 2D-nanofocusing processes on a chip [47]. To the further increase the field enhancement and localization, the $3 \mathrm{D}$ nanofocusing process is necessarily required. In recent, we have successfully demonstrated, experimentally, the highly efficient on-chip 3D metal-insulator-metal (MIM) nanofocusing structures [8]. We realized those structures on a chip by employing electron-beam-induced deposition (EBID)/ focused-ion beam (FIB) and demonstrated highly localized light confinement using the two-photon photoluminescence techniques. From the experimental measurements, we have observed the intensity enhancement of 400 within a $14-$ by- $80 \mathrm{~nm}^{2}$ cross-sectional area from a $200-$ by-500-nm ${ }^{2}$ area and the coupling efficiency of $-1.3 \mathrm{~dB}(74 \%$ transmittance).

In this work, we combine our previous fabrication/experimental work with in-depth theoretical design, analysis, and discussion to provide a detailed picture of the highly efficient, on-chip nanofocusing process which is linearly tapered in 3D. We highlight three major distinctive advantages of our MIM gap-plasmon nanofocusing device which we have theoretically understood and experimentally observed. (1) Hotspot size: Because we utilize the fundamental MIM anti-symmetric mode with no theoretical cutoff, it is possible to reduce the field profile down to the size of the MIM cross-section even in the deep sub-wavelength spaces. (2) Loss optimization: The precisely engineered, linear tapering geometry of our device effectively overcomes the major loss mechanisms present in the large wavevector region where the most power loss occurs during the nanofocusing process. (3) On-chip nanofabrication: Our 
process involves the use of EBID/FIB and standard IC processes that are highly controllable and compatible with other on-chip device fabrication techniques.

\section{THEORY}

\section{Fundamental Metal-Insulator-Metal (MIM) Waveguide Mode}

$\mathrm{An} \mathrm{Au-SiO2-Au} \mathrm{(metal-insulator-metal;} \mathrm{MIM)} \mathrm{surface-plasmon-polariton} \mathrm{(SPP)} \mathrm{guide} \mathrm{is}$ schematically presented in Fig. 1a. As the name "MIM" implies, the structural symmetry exists with respect to the mirror plane that cuts through the center of the $\mathrm{SiO}_{2}$ layer and is parallel to the $x-y$ plane. As a result, supported SPP modes in the guide can be classified into two major eigenstates [9]. One is symmetric (S), and the other is anti-symmetric (AS) modes. Figure 1b shows the existing modes supported by the proposed MIM-SPP waveguide structure with thickness $h$ between $50 \mathrm{~nm}$ and $300 \mathrm{~nm}$. The width of the waveguide $w$ is related to $h$ through the equation $w=(500 \mathrm{~nm} / 200 \mathrm{~nm}) \times h$, and the frequency of interest is $360 \mathrm{THz}$. In this figure, the fundamental AS mode (or AS1) does not exhibit any cut-off even for the sub-nanoscale dielectric thickness/width. So, we are most interested in the fundamental AS1 mode because it achieves the

a
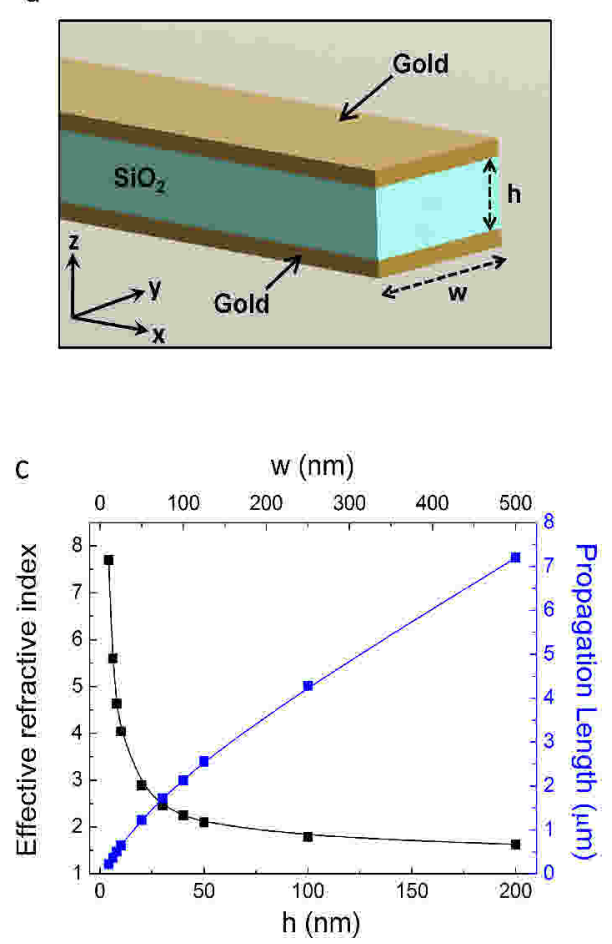

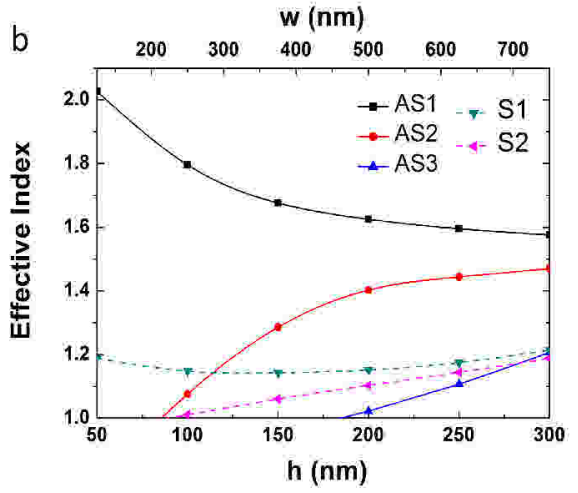

$d$

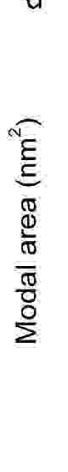

W (nm)

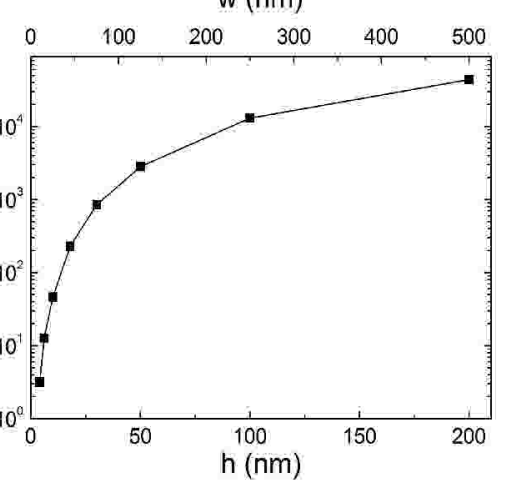

e

$h=200 \mathrm{~nm}$

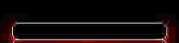

$\longrightarrow$

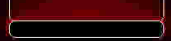

$\mathrm{h}=100 \mathrm{~nm}$

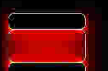

$\mathrm{h}=50 \mathrm{~nm}$

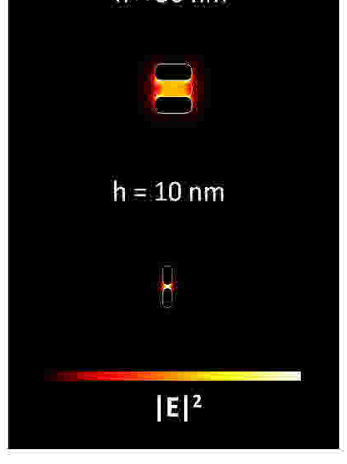

Fig. 1. a, Schematic diagram of the Au-SiO supported by the proposed MIM-SPP waveguide as a function of $\mathrm{SiO}_{2}$ thickness $(h)$. Here, "AS" and "S" represent the antisymmetric and symmetric modes, respectively. c, Effective index and propagation length of the fundamental anti-symmetric mode (AS1) as a function of $h$ and $w$. d, Effective modal area along the cross section in the MIM SPP waveguide as a function of $h$ and $w$. e, Cross-sectional side views of the $E^{2}$ profiles along the $y-z$ plane for $h=200,100,50$, and $10 \mathrm{~nm}$, respectively.

Figure 1c shows the effective refractive indices and the propagation lengths as a function of the $\mathrm{SiO}_{2}$ thickness $(h)$ and width $(w)$ of the fundamental AS1 mode. The effective index of refraction rapidly increases as the cross-sectional area $(w \times h)$ decreases because the smaller 
cross-sectional area of the insulator pushes the propagating waves deeper into the metal layer [9]. This deeper submersion of the propagating waves also causes the propagation length $\left(L_{m}\right)$ to decrease more rapidly due to the higher absorption loss in the metal. Figure 1d shows the effective modal areas $\left(A_{m}\right)$ of the AS1 mode as a function of $h$ and $w$. As the cross-sectional dimensions of the MIM-SPP waveguide are reduced, the size of the modal area decreases much more rapidly in a nonlinear manner. The intensity profiles of the electric field for $h=200,100$, 50, $10 \mathrm{~nm}$ are shown in Fig. 1e.

\section{Loss Minimization in MIM-SPP Nanofocusing}

The field of the fundamental anti-symmetric (AS) MIM-SPP mode can be focused down to a deep sub-wavelength space, as described in Fig.1, because they achieve very large wavevectors and effective refractive indices. To minimize the losses that occur during the nanofocusing process, it is essential to analyze the loss mechanisms in the large wave-vector regime because the losses that originate in the large wave-vector regime are most significant and makes much bigger contribution to the total loss. To simplify our theoretical treatment, we consider the 2-dimensional MIM SPP geometry shown in Fig. 2.
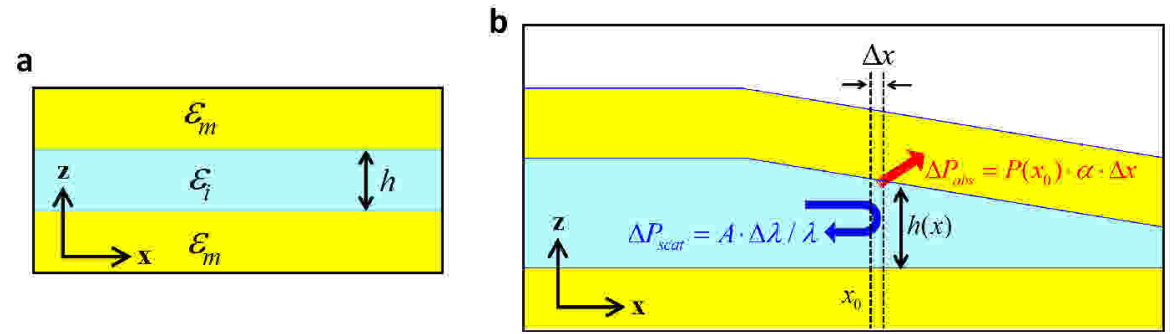

Fig. 2. a, Schematic of two-dimensional MIM SPP waveguide structure with parameters. b, Schematic of tapered structure. $\Delta P_{\text {scat }}$ and $\Delta P_{\text {abs }}$ represent generated scattering and absorption losses, respectively.

The loss $\left(\Delta P_{\text {loss }}\right)$ that occurs over a unit length $(\Delta x)$ along the propagation direction between $x=x_{0}$ and $x=x_{0}+\Delta x$ can be separated into two major parts, the scattering $\left(\Delta P_{\text {scat }}\right)$ and absorption $\left(\Delta P_{a b s}\right)$ terms, as shown in Fig. 2b. Under adiabatic conditions, the generated scattering loss $\Delta P_{\text {scat }}$ is governed by $\Delta \lambda / \lambda[10]$, where $\Delta \lambda$ represents the wavelength difference between $x=x_{0}$ and $x=x_{0}+\Delta x$. The absorption loss can be expressed as $\Delta P_{a b s}=P_{0} \alpha \Delta x$ where $P_{0}$ is the power at $x=x_{0}$ and $\alpha$ is an absorption coefficient (per unit length). Using these terms and the relation for $\alpha=\operatorname{Im}[k]$, the sum of the two major losses becomes

$$
\Delta P_{\text {loss }}=\Delta P_{\text {scat }}+\Delta P_{a b s}=A \cdot \Delta \lambda / \lambda+P_{0} \cdot \alpha \cdot \Delta x=A \cdot \Delta \lambda / \lambda+B \cdot \operatorname{Im}[k] \cdot \Delta x
$$

where $A$ and $B$ are constants.

Using the approximated dispersion relation in large- $k$ regime $(k \cdot h=f(\omega))$ as well as the relation $\Delta x \approx \lambda$ that originates from the large- $k$ approximation, Eq. (1) becomes

$$
\Delta P_{\text {loss }} \approx A \cdot(\Delta h / \Delta x)+B \cdot \operatorname{Im}[k] \cdot \lambda=C \cdot(\Delta h / \Delta x)+D
$$

where $C$ and $D$ are constants for a fixed frequency.

Equation (2) implies that the generated total loss $\left(\Delta P_{\text {loss }}\right)$ is minimized as the term $(\Delta h / \Delta x)$, which represents the slope of the taper, has to be constant or the tapering geometry is 
linear. Hence, employing an optimized linear taper in the MIM-SPP waveguide could significantly minimize the major losses that occur in a large- $k$ regime during nanofocusing.

\section{D MIM Nanofocusing}

Based on the theoretical prediction, we applied its concept to the 3D MIM nanofocusing structure, which is schematically presented in Fig. 3a-c. In the 3D tapering section, the ratio between the width $(w)$ and the height $(h)$ are kept constant, forming the linearly tapered geometry. Figure $3 \mathrm{~d}$ shows the coupling efficiency as a function of taper angle $(\alpha)$ for four sub-100nmscale waveguides with $h=30,15,5$, and $2 \mathrm{~nm}$. Regardless of the value of $h$, the highest coupling efficiency was calculated for $\alpha=20^{\circ}: 83 \%, 77 \%, 66 \%$, and $56 \%$ for $h=30,15,5$, and $2 \mathrm{~nm}$, respectively. For large $\alpha$, coupling was predominantly limited by the scattering due to the severely mismatched wave-vectors. On the other hand, small $\alpha$ was less than optimal, and coupling was limited by the absorption losses in the longer tapered section. The resistive and scattering losses in the tapered section were balanced for $\alpha$ between $15^{\circ}$ and $30^{\circ}$ in all four cases. Figure $3 \mathrm{e}$ shows the $E^{2}$ enhancement and the coupling efficiency at the junction between the tapered section and the sub-100nm-scale waveguide as a function of $h$ for $\alpha=20^{\circ}$. This figure shows that the maximum intensity was strongly enhanced for values of $h$ less than $10 \mathrm{~nm}$, even though the coupling loss increased significantly. This behavior resulted from the high spatial localization of the field and the increase in the effective refractive index. For a sub-100nm-scale waveguide, $2.0 \mathrm{~nm}$ tall and $5.0 \mathrm{~nm}$ wide, the maximum intensity enhancement was predicted to be as high as $\sim 3.0 \times 10^{4}$ at $\alpha=20^{\circ}$ with a coupling loss of $2.5 \mathrm{~dB}$.
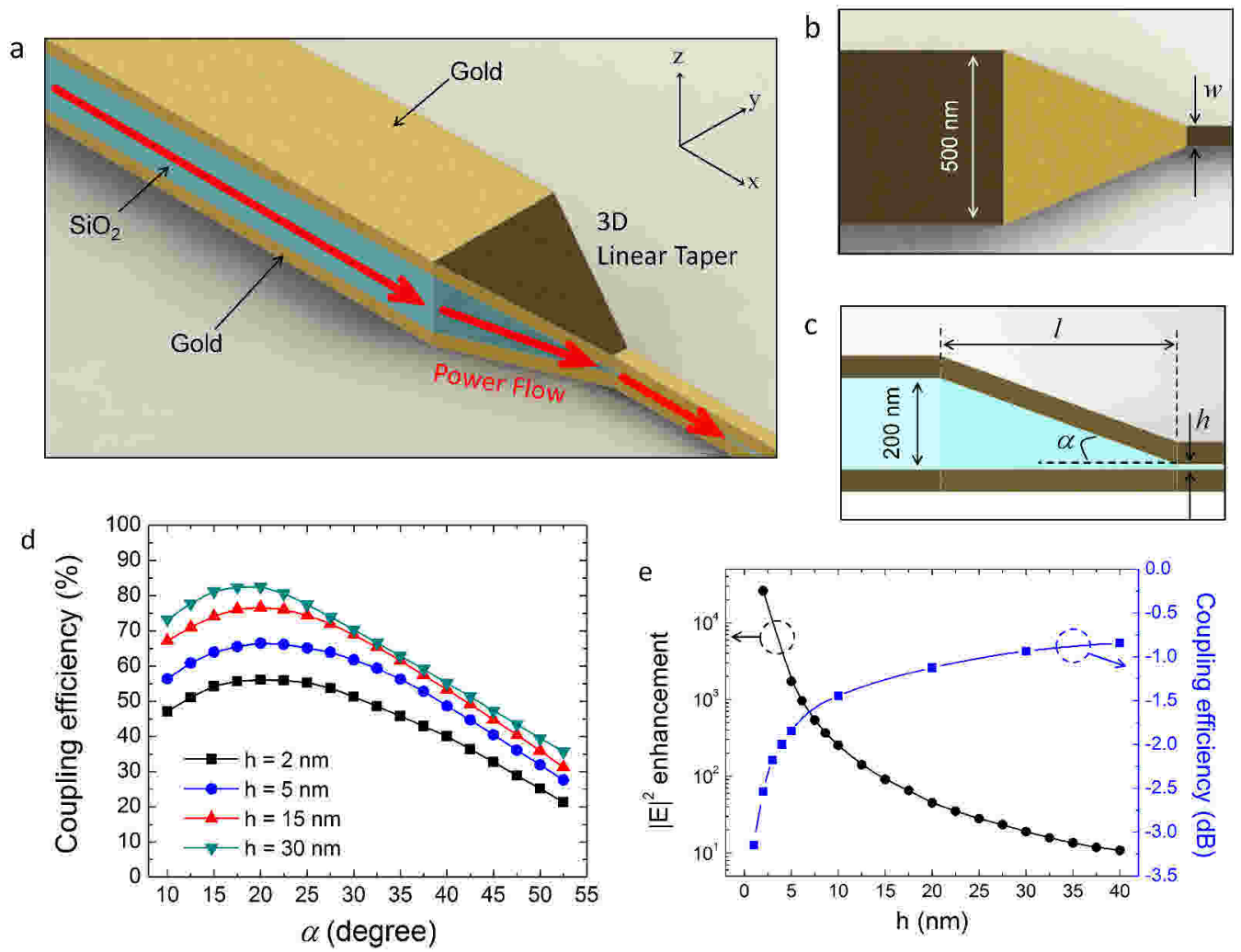

Fig. 3. a, b, c, Perspective, top, and side views, respectively, of a schematic illustration of a 3D nanofocusing structure,

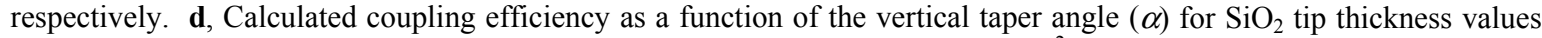
(h) of $30,15,5$, and $2 \mathrm{~nm}$ in the $3 \mathrm{D}$ nanofocusing structure with an infinite tip. e, $E^{2}$ enhancement and coupling efficiency on the $\mathrm{dB}$ scale as a function of $h$ for $\alpha=20^{\circ}$. 


\section{EXPERIMENTS}

Based on the optimal design, we fabricated a 3D MIM nanofocusing structure on a fused silica substrate using EBID, FIB, and e-beam evaporation [8]. Figure 4a shows the scanning electron microscopy (SEM) images of the fabricated 3D MIM nanofocusing structure. The minimum width and height of the intermediate dielectric $\left(\mathrm{SiO}_{2}\right)$ layer were measured as $w=80$ $\mathrm{nm}$ and $h=14 \mathrm{~nm}$.
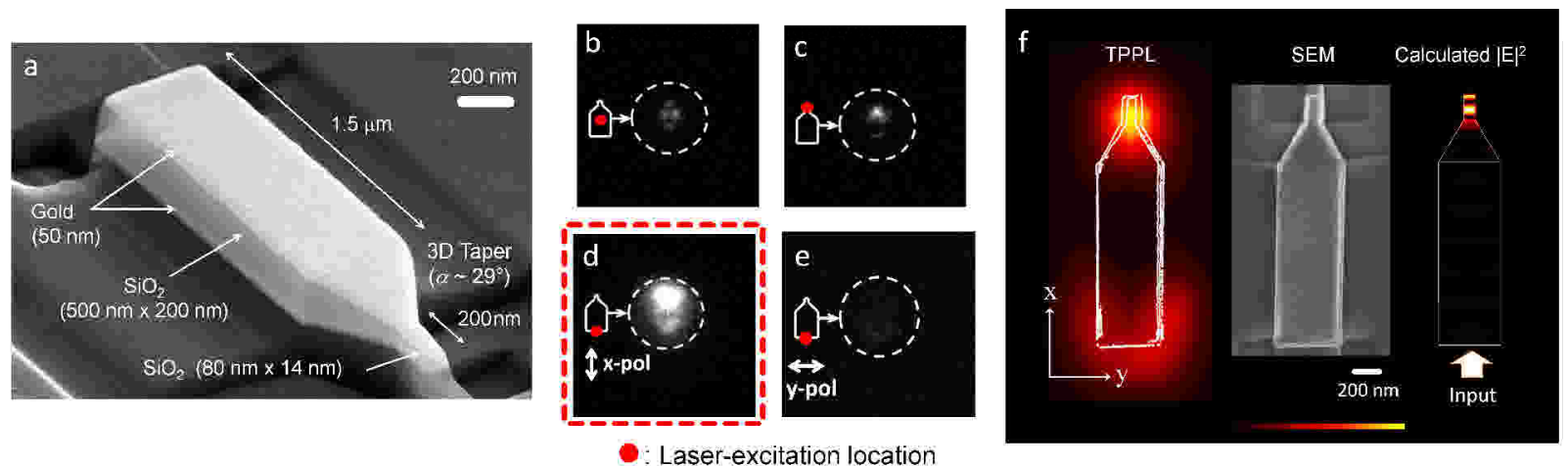

Fig. 4. a, SEM image of a fabricated 3D on-chip nanofocusing structure. b, c, d, EMCCD images of TPPL emission for four different excitation scenarios. The red dots indicate the positions of laser excitation. Here, the laser beams were $x$ polarized. e, TPPL image of the device for $y$-polarized excitation in the case shown in Figure 4d. f, The unsaturated intensity map of Figure $4 \mathbf{d}$ superimposed onto the contours of the SEM image of the device. Top view SEM image of the characterized sample and the simulated $|E|^{2}$ intensity.

Figure $4 \mathrm{~b}-\mathrm{d}$ shows the electron-multiplying charge-coupled-device (EMCCD) images of the two-photon photoluminescence (TPPL) emission profiles for three excitation scenarios. Here, the TPPL emission is generated in the gold layers on the sample, which intensity is very sensitive to the focused field strength at the nanofocusing device. For excitation, we delivered $830 \mathrm{~nm}$, $120 \mathrm{fs}$ Ti:sapphire laser pulses (54 MHz repetition rate) at an average power of $210 \mu \mathrm{W}$ to the back aperture of the sample. The position of laser excitation was controlled by moving the sample stage. The excitation light polarized along the $x$-axis ( $x$-polarization) clearly indicated that the maximum photon coupling into the tip was observed only when the light was properly coupled into the back aperture of the rectangular body, thereby generating SPPs that travelled through the 3D tapered coupler and concentrated in the tip (Fig. 4d). In the other three scenarios, the field enhancement at the tip was minimal (Fig. $4 \mathrm{~b}-\mathrm{c}$ ). Note that the $x$-polarized excitation light generated the desired transverse magnetic (TM) anti-symmetric plasmonic mode and expected enhancement in the MIM structure. Figure 4e shows a TPPL image of the device during excitation with $y$-polarized light at the same excitation location and power as in the $x$ polarization case shown in Fig. 4d. This clearly proved that the coupled mode responsible for the strong enhancement in the 3D nanofocusing structure shown in Fig. $4 \mathrm{~d}$ was the anti-symmetric TM plasmonic mode.

The location of the brightest TPPL emission spot in Fig. 4d was more accurately estimated by superimposing the unsaturated intensity map captured using the EMCCD camera onto the contour of the device's SEM image, as shown in Fig. 4f. This figure clearly showed that a maximum TPPL emission was observed in the tip. Weaker TPPL emission, observed at the location of the laser excitation (near the back aperture of the rectangular body), resulted from the local confinement at the edges. The rightmost figure in Fig. $4 \mathrm{f}$ shows the simulated $E^{2}$ profile with dimensions equal to those of the fabricated sample. The location of the maximum $E^{2}$ value 
in the simulation result agreed well with the location observed in the measured intensity map on the left; however, the nanoscale interference profile in the tip, as revealed by the simulation, could not be observed in the experimentally obtained TPPL intensity map due to the diffraction limit of the imaging system.

\section{DISCUSSION}

The extreme enhancement in the 3D MIM nanofocusing structure originated from its implemented tapering geometry. To demonstrate and quantify the intensity enhancement that originated from geometric effects, the strength of the TPPL emission from a nearby flat Au film, from which no significant field enhancements could be expected due to the surface geometry, was measured as a reference and then compared with that of the tip. The ratio between the TPPL intensities from the tip and the reference $\mathrm{Au}$ film was measured to be 1.8, where the average excitation powers incident on the nanofocusing structure and on the flat Au surface were $210 \mu \mathrm{w}$ and $4 \mathrm{~mW}$, respectively. Based on those values and the calculated effective TPPL areas, the intensity enhancement between the incident beam and the localized field at the tip was estimated at 125 . Finally, considering the input coupling efficiency of $\sim 15 \%$ between the incident beam and the MIM body waveguide and the effective modal areas, the intensity enhancement between the body and the tip was calculated to be $\sim 400$, which was in excellent agreement with the simulation result, 410. The coupling efficiency through the tapered section without the feedback effect was estimated to be $>-1.3 \mathrm{~dB}(74 \%)$.

\section{CONCLUSION}

We have demonstrated and analyzed a highly efficient on-chip 3D nanofocusing structure. We analytically reached the conclusion that the linearly MIM tapered geometry in onchip 3D MIIM nanofocusing structure could minimize the intrinsic losses which occur during nanofocusing. The optimized design was realized on a chip, and the implemented 3D on-chip nanofocusing structure showed highly localized light confinement. We strongly believe that this highly efficient on-chip 3D plasmonic nanofocusing approach will be useful in a variety of onchip nanoscale photonic/plasmonic applications.

\section{REFERENCES}

1. M. T. Hill et al., Nat. Photon. 1, 589-594 (2007).

2. L. Tang et al., Nat. Photon. 2, 226-229 (2008).

3. T. Nikolajsen, K. Leosson, and S. I. Bozhevolnyi, Appl. Phys. Lett. 85, 5833-5835 (2004).

4. E. Verhagen, L. Kuipers, and A. Polman, Nano Lett. 10, 3665-3669 (2010).

5. A. R. Davoyan et al., Phys. Rev. Lett. 105, 116804 (2010).

6. D. F. P. Pile and D. K. Gramotnev, Appl. Phys. Lett. 89, 04111 (2006).

7. V. S. Volkov et al., Nano Lett. 9(3), 1278-1282 (2009).

8. H. Choo et al., Nat. Photon. 6, 838-844 (2012).

9. D. F. P. Pile et al., Appl. Phys. Lett. 87, 261114 (2005).

10. Conway, Ph.D. thesis (University of California, Los Angeles, 2006). 